\title{
Coherent transfer of photoassociated molecules into the rovibrational ground state
}

\author{
K. Aikawa, ${ }^{1}$ D. Akamatsu, ${ }^{2}$ M. Hayashi, ${ }^{3}$ K. Oasa, ${ }^{1}$ J. Kobayashi, ${ }^{2}$ \\ P. Naidon, ${ }^{4}$ T. Kishimoto, ${ }^{5}$ M. Ueda, ${ }^{4,6}$ and S. Inouye ${ }^{2,4}$ \\ ${ }^{1}$ Department of Applied Physics, The University of Tokyo, Hongo, Bunkyo-ku, Tokyo 113-8656, Japar ${ }^{*}$ \\ ${ }^{2}$ Institute of Engineering Innovation, The University of Tokyo, Yayoi, Bunkyo-ku, Tokyo 113-8656, Japan \\ ${ }^{3}$ Department of Applied Physics, The University of Tokyo, Hongo Bunkyo-ku, Tokyo 113-8656, Japan \\ ${ }^{4}$ JST, ERATO, Yayoi, Bunkyo-ku, Tokyo 113-8656, Japan \\ ${ }^{5}$ Center for Frontier Science and Engineering, University of Electro-communications, Chofu, Tokyo 182-8585, Japan \\ ${ }^{6}$ Department of Physics, The University of Tokyo, Yayoi, Bunkyo-ku, Tokyo 113-8656, Japan
}

(Dated: July 20, 2018)

\begin{abstract}
We report on the direct conversion of laser-cooled ${ }^{41} \mathrm{~K}$ and ${ }^{87} \mathrm{Rb}$ atoms into ultracold ${ }^{41} \mathrm{~K}^{87} \mathrm{Rb}$ molecules in the rovibrational ground state via photoassociation followed by stimulated Raman adiabatic passage. High-resolution spectroscopy based on the coherent transfer revealed the hyperfine structure of weakly bound molecules in an unexplored region. Our results show that a rovibrationally pure sample of ultracold ground-state molecules is achieved via the all-optical association of laser-cooled atoms, opening possibilities to coherently manipulate a wide variety of molecules.
\end{abstract}

PACS numbers: $37.10 . \mathrm{Mn}, 33.15 . \mathrm{Pw}, 42.50 . \mathrm{Ct}, 82.80 . \mathrm{Ms}$

Progress in cooling and manipulating atoms has led to diverse applications. Even more possibilities will open up if molecules are cooled and prepared in the rovibrational ground state because then the translational, vibrational, and rotational degrees of freedom of the molecules can be manipulated with greater accuracy. The potential applications range from novel quantum phases to ultracold chemistry, quantum computation, and precision measurements [1]. Various schemes to achieve cold molecules have been demonstrated thus far. Direct cooling of molecules has been limited in terms of temperature [2]. Recently, quantum gases of molecules in the rovibrational ground state have been achieved [3 [5] with a coherent optical transfer of weakly bound molecules produced via the magnetoassociation [ [6] of quantum degenerate atoms. However, this scheme is only applicable to atomic species for which magnetoassociation is available. An alternative general method for producing weakly bound molecules from ultracold atoms is photoassociation (PA) 7]. Up to now, only the incoherent formation of molecules in the vibrational ground state via photoassociation has been reported [8-10]. Although the direct photoassociation yielded molecules in the $v^{\prime \prime}=0$, $J^{\prime \prime}=2$ level predominantly [10], the state-selective photoassociative formation of cold molecules in the rovibrational ground state is yet to be achieved.

Here, we report that a rotationally and vibrationally pure sample of ultracold ${ }^{41} \mathrm{~K}^{87} \mathrm{Rb}$ molecules in the rovibrational ground state could be directly produced in a magneto-optical trap (MOT) of ${ }^{41} \mathrm{~K}$ and ${ }^{87} \mathrm{Rb}$ via photoassociation followed by stimulated Raman adiabatic passage (STIRAP) [11]. In spite of the low laser intensity due to the large volume of our molecular gas, we could reach a STIRAP transfer efficiency of more than $70 \%$ by employing an intermediate state with a narrow natural linewidth. In order to identify the two-photon reso- nance precisely, we developed a new detection scheme, spontaneous-decay-induced double resonance (SpIDR), which provided the high-resolution spectra of one-photon transitions from PA molecules and allowed us to observe a two-photon dark resonance [12]. Our results show that a rovibrationally pure sample of ultracold molecules in the ground state is achieved via the all-optical association of laser-cooled atoms, opening novel possibilities to coherently manipulate a wide variety of cold molecules that can be produced via photoassociation.

Fig. [1]shows the schematic representation of the coherent transfer of ultracold ${ }^{41} \mathrm{~K}^{87} \mathrm{Rb}$ molecules. The potential curves are taken from Ref. 13. Details of the experimental setup for the production and detection of weakly bound molecules is found elsewhere [14]. For the present study, we used ${ }^{41} \mathrm{~K}^{87} \mathrm{Rb}$ molecules in the $v^{\prime \prime}=91, J^{\prime \prime}=0$ level of $X^{1} \Sigma^{+}$produced via photoassociation of ${ }^{41} \mathrm{~K}$ and ${ }^{87} \mathrm{Rb}$ atoms in a MOT. The typical numbers, densities and temperatures of atoms were $1 \times 10^{8}, 2 \times 10^{11} \mathrm{~cm}^{-3}$ and $400 \mu \mathrm{K}$ for ${ }^{41} \mathrm{~K}$ and $2 \times 10^{8}, 4 \times 10^{11} \mathrm{~cm}^{-3}$ and $100 \mu \mathrm{K}$ for ${ }^{87} \mathrm{Rb}$, respectively. The PA laser resonant to the $J^{\prime}=1$ level of $\Omega=0^{+}$(wave number $\sim 12570$ $\mathrm{cm}^{-1}$, intensity $\sim 1 \times 10^{3} \mathrm{~W} \mathrm{~cm}^{-2}$ ) was applied for 10 ms. Molecules were ionized through resonance enhanced multi-photon ionization (REMPI) by a pulsed dye laser and detected by micro-channel plates (MCP). The entire experimental procedure was operated at $9 \mathrm{~Hz}$.

Despite the successful demonstrations on quantum gases, the STIRAP transfer into the rovibrational ground state was not straightforward for PA molecules. Any residual magnetic field could cause decoherence for Raman coupling between two molecular states since our molecular sample was not spin-polarized. In addition, untrapped, high-temperature PA molecules occupied a volume larger $\left(\sim 1 \mathrm{~mm}^{3}\right)$ than that occupied by the trapped quantum gases $\left(\sim 10^{-4} \mathrm{~mm}^{3}\right)$. This limited the 


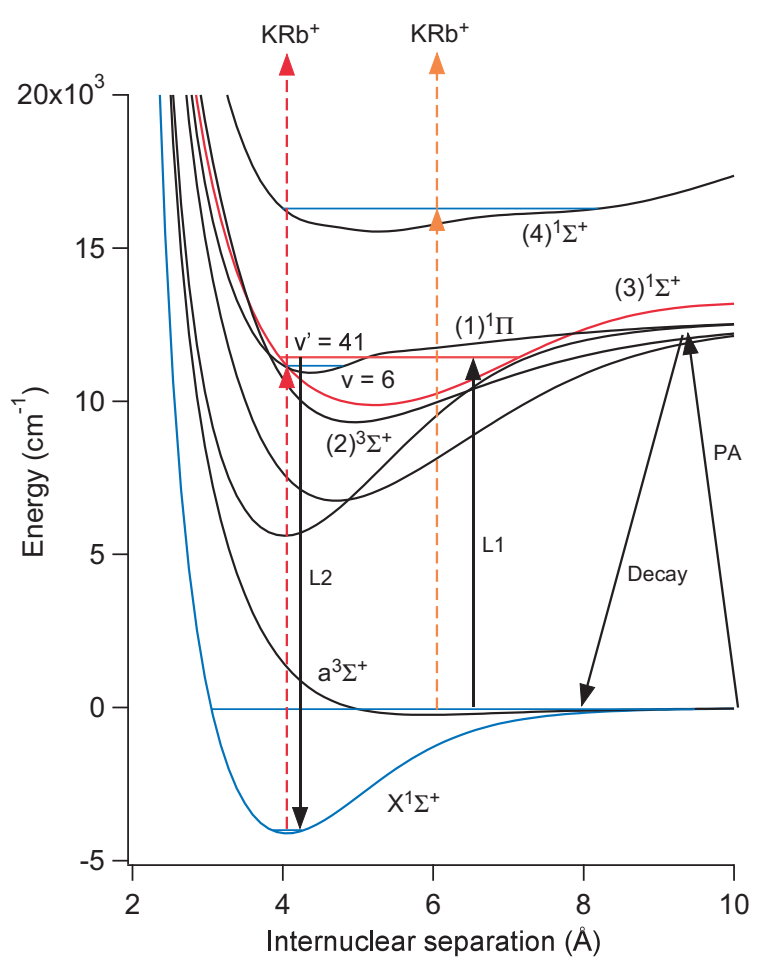

FIG. 1. Relevant molecular potential energy curves of KRb. Weakly bound molecules were produced by the photoassociation of laser-cooled ${ }^{41} \mathrm{~K}$ and ${ }^{87} \mathrm{Rb}$ atoms. Molecules in the $v^{\prime \prime}=91, J^{\prime \prime}=0, F^{\prime \prime}=2$ level of $X^{1} \Sigma^{+}$were coupled to the $v^{\prime \prime}=0, J^{\prime \prime}=0$ level of $X^{1} \Sigma^{+}$by a two-photon transition with wavelengths near $875(\mathrm{~L} 1)$ and 641 (L2) $\mathrm{nm}$ via the $v^{\prime}=41, J^{\prime}=1$ level of $(3)^{1} \Sigma^{+}$. For depletion spectroscopy and SpIDR spectroscopy, two-photon ionization through the $(4)^{1} \Sigma^{+}$state was used. For the detection of the rovibrational ground-state molecules, a three-photon transition mediated by the $v^{\prime}=6$ level of $(1)^{1} \Pi$ was used.

available laser intensity for driving the transition.

These difficulties were circumvented by starting from singlet molecules which was insensitive to magnetic fields. Utilizing singlet excited states with a narrow natural linewidth as an intermediate state, we realized an efficient Raman transfer of PA molecules into a singlet ground state. This was in contrast with previous works on the two-photon transfer of heteronuclear molecules [3, 8], which started from triplet molecules and transferred the PA molecules into the singlet ground state via the $\Omega=1$ state (the spin-mixed state of $(2)^{3} \Sigma^{+}$, $(1)^{3} \Pi$ and $\left.(1)^{1} \Pi\right)$. A transition strength comparable to the $\Omega=1$ state was obtained with the $v^{\prime}=41$ level of $(3)^{1} \Sigma^{+}$[14, 15], whereas the narrow natural linewidth of this state $(\Gamma \sim 2 \pi \times 300 \mathrm{kHz}$, calculated from Ref. 16) allowed us to obtain an efficient transfer with a relatively low laser intensity.

Our optical system for the Raman transition consisted of four lasers. Two diode lasers $(875 \mathrm{~nm}$ and $641 \mathrm{~nm})$ were locked to an ultra-low expansion (ULE) cavity with a dual-wavelength coating. These lasers worked as mas-
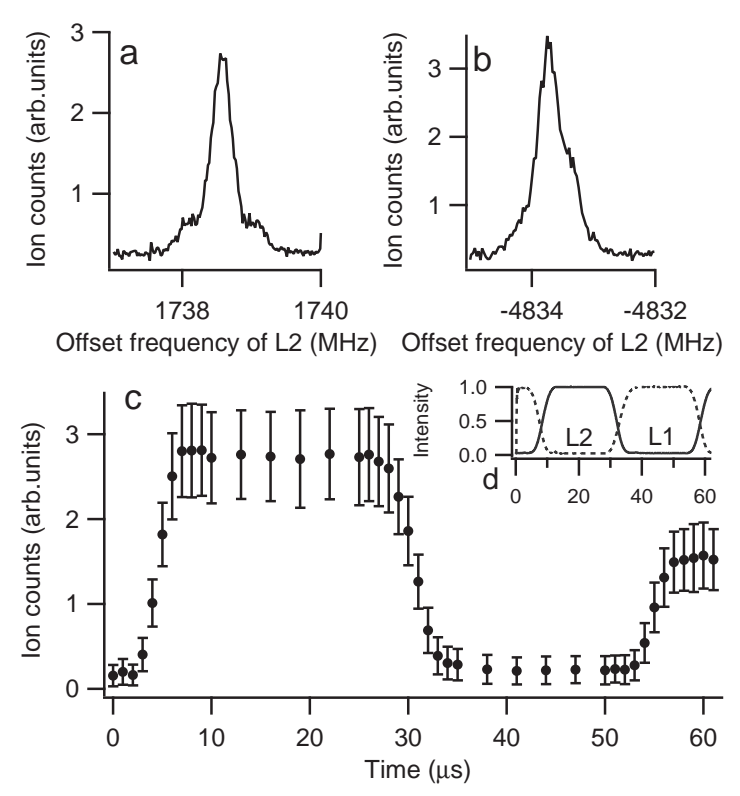

FIG. 2. STIRAP transfer from the $v^{\prime \prime}=91, J^{\prime \prime}=0, F^{\prime \prime}=2$ level to the $v^{\prime \prime}=0$ level of $X^{1} \Sigma^{+}$. (a) Transfer to the $J^{\prime \prime}=0$ level. The population in the $v^{\prime \prime}=0$ level of $X^{1} \Sigma^{+}$is plotted against the frequency of the down transition laser (L2). (b) Transfer to the $J^{\prime \prime}=2$ level. The asymmetry observed in the $J^{\prime \prime}=2$ spectrum is expected to be a result of the hyperfine structure induced by the nuclear electric quadrupole moments of ${ }^{87} \mathrm{Rb}$. (c) Time evolution of the population in the $v^{\prime \prime}=0$, $J^{\prime \prime}=0$ level of $X^{1} \Sigma^{+}$during the multiple STIRAP transfer process. The error bars indicate the standard deviation. (d) Time variation of the intensities of the Raman lasers during the multiple-transfer process. Both intensities are normalized to unity. During the waiting time of $20 \mu$ s set between the transfers, the remaining population that is not transferred by STIRAP is completely removed by the resonant Raman lasers.

ter lasers. Two slave lasers, a ring dye laser (641 nm) and a diode laser $(875 \mathrm{~nm})$, were then locked to master lasers through an optical phase-locked loop (OPLL) [17] and used for experiments. The scanning of the Raman lasers was realized by sweeping the microwave source for OPLL. In this report, the microwave frequency for OPLL was noted as an offset frequency. The typical short-term linewidth of the Raman lasers was less than $10 \mathrm{~Hz}$, whereas the long-term stability of the ULE cavity was estimated to be less than $100 \mathrm{kHz}$ by monitoring the Cs D2 transition. The $1 / e^{2}$ diameter of the Raman lasers was set as $1.5 \mathrm{~mm}$ which was larger than the size of atomic clouds. For the STIRAP transfer, the power of the laser L1 and the laser L2 was set to $9 \mathrm{~mW}$ and 25 $\mathrm{mW}$, respectively.

Fig. 2 a shows the population in the $v^{\prime \prime}=0$ level of $X^{1} \Sigma^{+}$after the STIRAP transfer into the $J^{\prime \prime}=0$ level as a function of the frequency of the down transition laser (L2). The observed two-photon linewidth of 200 $\mathrm{kHz}$ was consistent with a simple three-level theoreti- 


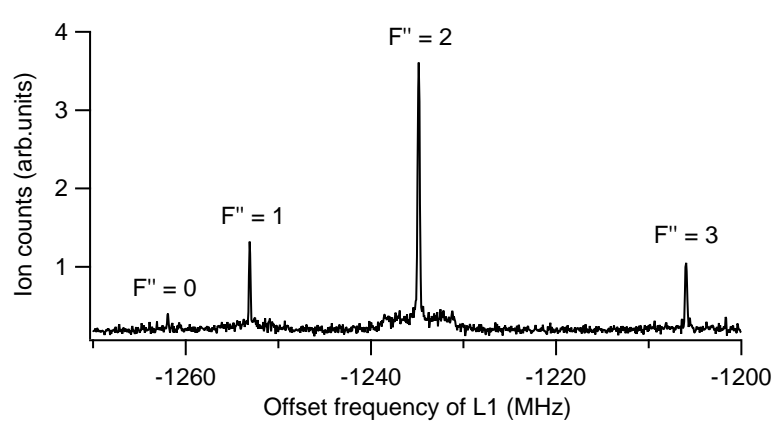

FIG. 3. STIRAP spectroscopy of weakly bound molecules (the $v^{\prime \prime}=91, J^{\prime \prime}=0$ level of $X^{1} \Sigma^{+}$). An efficient transfer into the rovibrational ground state enables the precision spectroscopy of the hyperfine structure of photoassociated molecules in an unexplored region with a typical accuracy of $10 \mathrm{kHz}$. The labeling is based on the total angular momentum including the nuclear spin.

cal model [11] when we took into account the Rabi frequency $(2 \pi \times 400 \mathrm{kHz})$ and the transfer duration $(10 \mu \mathrm{s})$. In order to confirm that Fig. 2 2 a indicated the rovibrational ground state, we also performed a STIRAP transfer into the $v^{\prime \prime}=0, J^{\prime \prime}=2$ level (Fig. 2b) and determined the rotational constant to be 1095.4(1) $\mathrm{MHz} \times h$ ( $h$ is the Planck's constant). The obtained value was in good agreement with the calculated value of 1095.362(5) $\mathrm{MHz} \times h$ obtained from the mass-scaling of the experimental value for ${ }^{40} \mathrm{~K}^{87} \mathrm{Rb}$ [18]. The typical number, density and temperature of the produced ground-state molecules were $10^{3}, 10^{6} \mathrm{~cm}^{-3}$, and $130 \mu \mathrm{K}$, respectively. The production rate in the rovibrational ground state was as high as $10^{4} \mathrm{~s}^{-1}$.

The direct evidence of the STIRAP transfer was obtained by studying the time evolution of the population in the ground state (Fig. 2r) during the multiple transfer process (Fig. 2 $\mathrm{d}$ ). The ground-state molecules after a single transfer were used for measuring the efficiency of transfer. We typically retrieved $53 \%$ of the groundstate molecules after the double-transfer process, which implied a single-step efficiency of $73 \%$. Currently, the efficiency was limited by the difference in the Doppler shift for the two lasers.

The STIRAP transfer into the rovibrational ground state can be used as new precision spectroscopy for weakly bound molecules in an unexplored region (Fig. 3). By mapping the population in the initial hyperfine levels onto the ground state, we could measure the hyperfine splitting of PA molecules with an accuracy of $10 \mathrm{kHz} \times h$. A higher resolution could be achieved by using a longer transfer duration and smaller intensities for the Raman lasers. The observed energy levels of the $F^{\prime \prime}=3,2,1$ levels with respect to the $F^{\prime \prime}=0$ level were $55.960(9)$, 27.089(7), and 8.852(7) $\mathrm{MHz} \times h$, respectively. The values of these energy levels as obtained by a coupled channel calculation were $56.180,27.176$, and $8.870 \mathrm{MHz} \times h$; these values were in reasonable agreement with our observations.

The rest of this report is devoted to SpIDR spectroscopy, which is an essential improvement of the onephoton spectroscopy of PA molecules. This improvement was necessary for identifying the structure of the excited states and observing a two-photon dark resonance. To date, depletion spectroscopy has been widely used for analyzing the transition from PA molecules [15]. A continuous-wave (CW) laser depletes the number of PA molecules, while the loss is monitored with REMPI. Although this spectroscopy was straightforward, we encountered a serious problem related to the signal-to-noise ratio when we attempted to record data at a low intensity in order to avoid saturation broadening. At a relatively high intensity, where we could observe a large signal, the spectral width broadened to tens of megahertz, washing out all the structure from the hyperfine levels. In addition, saturation broadening smoothed out the transparency peak of the two-photon dark resonance and made it difficult to identify the two-photon resonance.

In order to improve the signal-to-noise ratio of depletion spectroscopy, we developed a method to monitor not the depletion but the creation of molecules in the excited state. In practice, we monitored the population in one of the vibrational levels in the ground state to which a significant fraction of excited molecules decay. Thus, we could obtain a background-free spectrum, thereby increasing the signal-to-noise ratio to observe the narrow linewidth of the $(3)^{1} \Sigma^{+}$state.

Fig. 4 4 shows the SpIDR spectrum of the $v^{\prime}=41$, $J^{\prime}=1$ level of $(3)^{1} \Sigma^{+}$. A considerably smaller power $(5$ $\mu \mathrm{W})$ and shorter time $(400 \mu \mathrm{s})$ than those required for depletion spectroscopy (>100 $\mu \mathrm{W}, 10 \mathrm{~ms}$ ) were sufficient to detect the excitation. This led to a significantly narrow linewidth close to the natural linewidth. The coarse splitting resulted from the hyperfine structure in the ground state, whereas the fine splitting implied the existence of the hyperfine structure in the excited state. For the sake of comparison, the depletion spectrum in the same frequency range is shown in Fig. $4 \mathrm{~b}$.

The SpIDR spectroscopy enabled us to observe a twophoton dark resonance (Fig. 45). The dip indicated the emergence of the two-photon dark state with the laser L2 12. By comparing the two frequencies at the dip, we determined the binding energy of the $v^{\prime \prime}=0, J^{\prime \prime}=0$ level of $X^{1} \Sigma^{+}$with respect to the $v^{\prime \prime}=91, J^{\prime \prime}=0$ level of $X^{1} \Sigma^{+}$ to be $-124955.92(4) \mathrm{GHz} \times h$. Taking into account the binding energy of the $v^{\prime \prime}=91, J^{\prime \prime}=0$ level with respect to the threshold $F_{\mathrm{K}}=1+F_{\mathrm{Rb}}=1,-374.75(3) \mathrm{GHz} \times h$, the binding energy of the $v^{\prime \prime}=0, J^{\prime \prime}=0$ level with respect to the atomic level without the hyperfine structure was determined to be $-125335.11(5) \mathrm{GHz} \times h$. From the width of the transparency peak, we determined the transition dipole moment for the down transition to be 0.0098(7)ea $e a_{0}$, assuming the natural linewidth of the ex- 

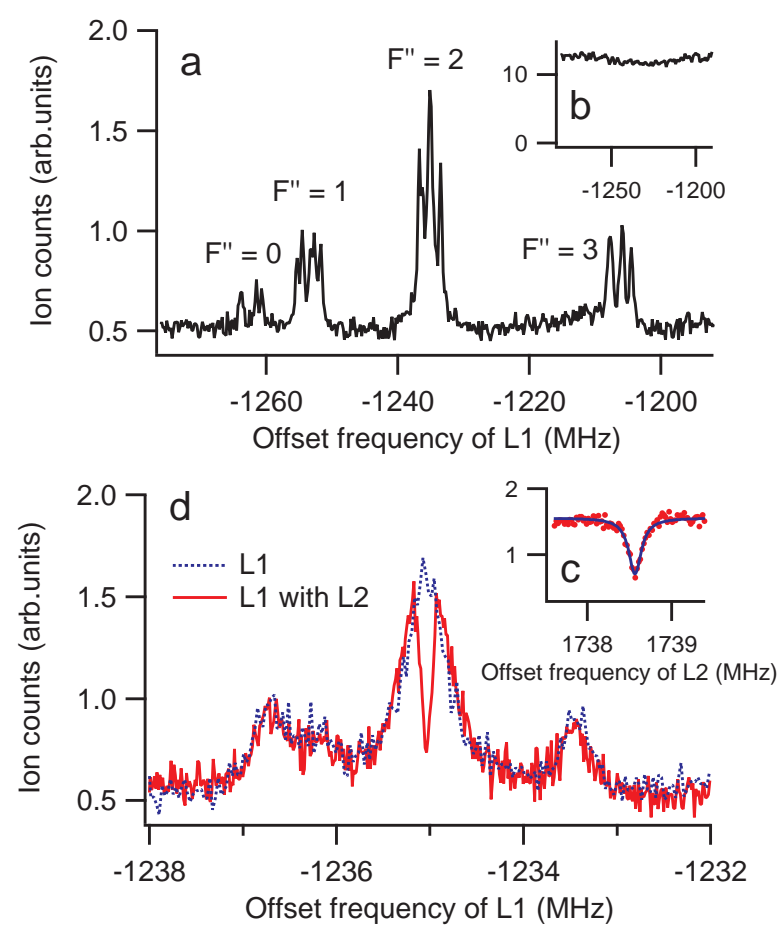

FIG. 4. SpIDR spectroscopy of photoassociated molecules. (a) SpIDR spectrum of the $v^{\prime}=41, J^{\prime}=1$ level of $(3)^{1} \Sigma^{+}$ taken by exciting molecules in the $v^{\prime \prime}=91, J^{\prime \prime}=0$ level of $X^{1} \Sigma^{+}$. Molecules formed by spontaneous decays from the excited state are counted against the offset frequency of the laser L1. The coarse splitting results from the hyperfine structure of the $v^{\prime \prime}=91, J^{\prime \prime}=0$ level of $X^{1} \Sigma^{+}$. The fine splitting in each line implies the existence of hyperfine structure in the excited state. For the two-photon transition, the largest peak $\left(F^{\prime \prime}=2\right)$ is employed as an initial state. (b) Depletion spectrum. In order to observe any signal, we had to increase the laser intensity by three orders of magnitude as compared to (a). Transitions from the $v^{\prime \prime}=91, J^{\prime \prime}=0$ level are barely observable since the ion counts are dominated by molecules in the $v^{\prime \prime}=91, J^{\prime \prime}=2$ level. (c) Two-photon dark resonance between the $v^{\prime \prime}=91, J^{\prime \prime}=0, F^{\prime \prime}=2$ level and the $v^{\prime \prime}=0$, $J^{\prime \prime}=0$ level of $X^{1} \Sigma^{+}$. The down transition laser (L2) is scanned while the up transition laser (L1) is held on resonance. The dots indicate experimentally obtained data and the solid curve is a fit to the data points. (d) Two-photon dark resonance obtained by scanning the laser L1. The laser L2 is held on resonance. The blue dashed curve indicates a SpIDR spectrum for the $F^{\prime \prime}=2$ level, while the red solid one indicates a spectrum obtained with the laser L2.

cited state to be $2 \pi \times 300 \mathrm{kHz}$. When we locked the laser L2 to the dip frequency and scanned the laser L1, we obtained a well-known dark resonance spectrum (Fig. 4d).

In conclusion, we realized an efficient STIRAP transfer of PA molecules into the rovibrational ground state. High-resolution spectroscopy of the hyperfine structure of PA molecules in an unexplored region was demonstrated using coherent transfer. We developed a highly sensitive detection scheme which enabled us to take a high-resolution spectrum of one-photon transitions and observe a two-photon dark resonance for PA molecules. Our method is readily extended to other atomic species for which laser cooling and photoassociation is available. Trapping molecules into an optical dipole trap [19] is the next key issue. The trapped sample of molecules will constitute a test tube for ultracold chemistry. Moreover, the high repetition rate makes our method a promising candidate for high-precision spectroscopy. The precision measurement of the electron-to-proton mass ratio in cold molecules [20] is within reach with our methods.

We thank M. Kozuma for helpful discussions, and M. Falkenau, Y. Tanooka, and K. Mori for experimental assistance in the early stages of the experiment. K. A. and D. A. acknowledge support from the Japan Society for the Promotion of Science.

* ka_cypridina@atomtrap.t.u-tokyo.ac.jp

[1] R. V. Krems, W. C. Stwalley, and B. Friedrich, Cold molecules: theory, experiment, applications (CRC, 2009); L. D. Carr, D. DeMille, R. V. Krems, and J. Ye, New J. Phys., 11, 055049 (2009).

[2] J. D. Weinstein et al., Nature, 395, 148 (1998); H. L. Bethlem, G. Berden, and G. Meijer, Phys. Rev. Lett., 83, 1558 (1999); M. S. Elioff, J. J. Valentini, and D. W. Chandler, Science, 302, 1940 (2003); M. Gupta and D. Herschbach, J. Phys. Chem. A, 103, 10670 (1999); S. A. Rangwala et al., Phys. Rev. A, 67, 043406 (2003).

[3] K.-K. Ni et al., Science, 322, 231 (2008).

[4] F. Lang et al., Phys. Rev. Lett., 101, 133005 (2008).

[5] J. G. Danzl et al., Nat. Phys., 6, 265 (2010).

[6] T. Kohler, K. Goral, and P. S. Julienne, Rev. Mod. Phys., 78, 1311 (2006).

[7] K. M. Jones, E. Tiesinga, P. D. Lett, and P. S. Julienne, Rev. Mod. Phys., 78, 483 (2006).

[8] J. M. Sage, S. Sainis, T. Bergeman, and D. DeMille, Phys. Rev. Lett., 94, 203001 (2005).

[9] M. Viteau et al., Science, 321, 232 (2008).

[10] J. Deiglmayr et al., Phys. Rev. Lett., 101, 133004 (2008).

[11] K. Bergmann, H. Theuer, and B. W. Shore, Rev. Mod. Phys., 70, 1003 (1998); N. V. Vitanov, M. Fleischhauer, B. W. Shore, and K. Bergmann, Adv. At. Mol. Opt. Phys., 46, 55 (2001); P. Kral, I. Thanopulos, and M. Shapiro, Rev. Mod. Phys., 79, 53 (2007).

[12] S. E. Harris, Phys. Today, 50, 36 (1997); M. D. Lukin, Rev. Mod. Phys., 75, 457 (2003); M. Fleischhauer, A. Imamoglu, and J. P. Marangos, ibid., 77, 633 (2005).

[13] S. Rousseau, A. R. Allouche, and M. Aubert-Frecon, J. Mol. Spectrosc., 203, 235 (2000).

[14] K. Aikawa et al., New J. Phys., 11, 055035 (2009).

[15] D. Wang et al., Phys. Rev. A, 75, 032511 (2007).

[16] R. Beuc et al., J. Phys. B, 39, S1191 (2006).

[17] L. Ricci et al., Opt. Comm., 117, 541 (1995).

[18] S. Ospelkaus et al., Phys. Rev. Lett., 104, 030402 (2010).

[19] E. R. Hudson et al., Phys. Rev. Lett., 100, 203201 (2008).

[20] T. Zelevinsky, S. Kotochigova, and J. Ye, Phys. Rev. Lett., 100, 043201 (2008); D. DeMille et al., ibid., 100, 043202 (2008). 